\title{
Docencia inversa aplicada a disciplinas jurídicas. Aplicación práctica en la asignatura de Derecho del Trabajo en el grado en ADE.
}

\section{Carlos García Gallego}

\begin{abstract}
La clase inversa, es una metodología docente en la que el profesor, con carácter previo a la exposición en clase de los temas correspondientes, pone a disposición del alumno los materiales de clase (en gran medida en formato audio visual) de modo que, vistos por éste, la clase presencial se convierte en una forma de repasar dichos contenidos y ponerlos en práctica mediante los ejercicios oportunos.

Esta metodología se ha puesto en práctica en la Universitat Politécnica de Valencia de manera oficial, mediante la creación de dos grupos específicos en el grado en Administración y Dirección de Empresas que se imparte en la Facultad de ADE y en el Grado de Ingeniería Informática de la ETSII.

Una de las asignaturas que ya se ha impartido en el primer cuatrimestre del presente curso académico 2014-2015, dentro del grado de ADE, siguiendo la citada metodología docente es Derecho del Trabajo.

En la presente comunicación se tratará de analizar el desarrollo y los resultados obtenidos en el grupo de docencia inversa de dicha asignatura, para finalmente concluir si la metodología utilizada en la misma puede ser extensible a otras asignaturas de contenido jurídico, y en caso afirmativo, en qué términos convendría hacerse.
\end{abstract}

\section{Keywords:}

Docencia inversa, Clase inversa,Flip-teaching, Flippedclassrom, Derecho, Derecho del Trabajo, Derecho Laboral, Jurídico. 
Docencia inversa aplicada a disciplinas jurídicas. Aplicación práctica en la asignatura de Derecho del Trabajo en el grado en $\mathrm{ADE}$.

\section{Introducción}

\subsection{Concepto de docencia inversa o flipped classroom}

No es objeto de este trabajo realizar un análisis pormenorizado del concepto de docencia inversa, flipteaching o flippedclassroom. No obstante, interesa resaltar brevemente en qué consisten los aspectos fundamentales de este modelo pedagógico puesto que en él se basa el conjunto del presente estudio.

La clase inversa es, como se ha dicho, un modelo pedagógico que se asienta sobre el trabajoautónomo que debe llevar a cabo el alumno a partir del material que le proporciona el profesor con carácter previo a la clase presencial. De ese modo, el profesor debe, durante el tiempo en clase, facilitar que los alumnos repasen, analicen, pongan en práctica y amplíen los conoicimiento trabajados previamente en casa.

En otras palabras, la docencia inversa implica una parte no presencial en la que los alumnostrabajan sobre el material puesto a disposición por el docente, y una parte presencial en la que se pone en práctica, con la ayuda del profesor, los contenidos trabajados en casa.

Por todo lo tanto, puede concluirse que esta metodología docente se apoya sobre dos columnas que deben gestionarse adecuadamente por el profesor. Por un lado, el diseño del material que debe ser puesto a disposición del estudiante y por otro, el diseño de las actividades que se realizarán en aula para reforzar y poner en práctica los contenidos puestos a disposición del alumno.

En cuanto a la preparación del material docente, sin descartar la utilización de diversas fuentes de contenidos, es muy recomendable la creación de material audiovisual (videos, screencast, polimedia...) puesto que es mucho más eficiente para el aprendizaje que el texto escrito y al mismo tiempo es aceptado por el estudiante de manera mucho más fácil. Piénsese, por ejemplo, que el alumno podrá visionar cuantas veces considere necesario los contenidos puestos a su disposición, lo que por sí sólo ya supone ampliar las posibilidades de comprensión y análisis de aquéllos.

Es muy importante a la hora de diseñar los materiales de estudio que estos sean claros y dirigidos específicamente a alcanzar el objetivo de aprendizaje previsto por el docente. En este sentido, se recomienda que el material audiovisual confeccionado no tenga una duración superior a los 10 minutos (por cada video) y que el número de videos o materiales que deba trabajar el estudiante antes de cada sesión no sea excesivo para evitar que se sature y pierda de vista el objetivo principal que debía trabajarse. 
Finalmente, es fundamental que el profesor diseñe un procedimiento de control del trabajo no presencial del alumno, mediante preguntas de test o la realización de ejercicios sencillos, para obligar al estudiante a trabajar esos contenidos que más tarde se aplicarán en la clase presencial. Este aspecto resulta fundamental para el éxito de la implantación de la docencia inversa, puesto que si no se consigue el método fracasa.

La segunda tarea del profesor en que se apoya la flippedclassroom sería el diseño de las actividades en el aula.

Como ya se ha dicho, no podrá tener éxito esta fase si previamente el estudiante no ha trabajado los materiales previstos por el docente para cada sesión presecial. Y esto depende de dos factores, la calidad (sencillez, utilidad, atractivo...) de los materiales puestos a disposición del alumno y el sistema de control diseñado para garantizar que dichos materiales se hayan trabajado previamente de forma autónoma.

El trabajo en el aula consistirá en poner en práctica los conocimientos adquiridos a través de los materiales de que dispone el alumno. Lo supone la realización de casos o problemas, de ejercicios, trabajos en grupo o actividades de laboratorio de manera que se adquieran o perfeccionen competencias tales como la capacidad de análisis, la cretividad o la mejora en la toma de decisiones.

Todos estos principios son los que, con mayor o menor fortuna, se han tratado de aplicar en la asignatura Derecho del Trabajo que se imparte durante el primer cuatrimestre en segundo curso del grado en ADE.

\subsection{Situación de partida.}

En el presente curso académico 2014-2015 se ha puesto en marcha una experiencia piloto, por iniciativa del Vicerrectorado de las Tecnologías de la Información y las Comunicaciones de la Universitat Politécnica de Valencia, consistente en la creación de dos grupos específicos de docencia inversa. Uno de ellos se ubica en el segundo curso del grado en Administración y Dirección de Empresas que se imparte en la Facultad de ADE y el otro, en el segundo curso del Grado de Ingeniería Informática de la Escuela Técnica Superior de Ingeniería Informática.

Se trata como se ha dicho, de grupos específicos de flippedclassroom, al estilo de los grupos ARA, en los que toda la docencia que en ellos se imparte debe hacerse siguiendo dicho modelo pedagógico. Además, teniendo en cuenta de que se trata una experiencia piloto de la que se quieren extraer conclusiones para valorar su posible aplicación de forma generalizada, dichos grupos se han configurado manteniendo un número reducido de alumnos (con un máximo de 20 o 25 matriculados),

\section{(cc) EY-NC-ND 2015, UniversitatPolitècnica de València}


Docencia inversa aplicada a disciplinas jurídicas. Aplicación práctica en la asignatura de Derecho del Trabajo en el grado en $\mathrm{ADE}$.

Atendiendo concretamente al grupo de docencia inversa de la facultad de ADE que se puso en marcha durante el primer cuatrimestre, conviene resaltar que se planteó la posibilidad de que los propios profesores de cada una de las asignaturas que configuraban ese grupo de flippedclassroom participara en la confección del horario especifico de dicho grupo decidiendo qué parte del conjunto de su asignatura iba a ser presencial y qué parte quedaría como no presencial.

La mayoría de los profesores decidió que la totalidad de la teoría de aula que les correspondía según el plan de estudios formaría parte de la docencia no presencial, quedando como trabajo en clase el tiempo correspondiente a las prácticas de aula, las prácticas informáticas y loss seminarios de aula.

La única excepción a este criterio se produjo en la asignatura Derecho del Trabajo (precisamente la que es objeto de este estudio) en la que, por las razones que más adelante se dirá, la parte no presencial no alcanza al $100 \%$ de la teoría de aula sino al $50 \%$ de este. Lo que se traduce en que la parte presencial es mayor que en el resto de asignaturas que configuran el grupo de flipteaching.

En cuanto a los datos de matrícula de este grupo específico, ya se ha mencionado que la idea era configurar un grupo con un máximo de 20 alumnos, teniendo en cuenta el carácter de experiencia piloto que se le había conferido. No obstante, la mayoría de las asignaturas de este grupo no alcanzaron siquiera los 10 alumnos matriculados, probablemente por el escaso tiempo que hubo para darlo a conocer entre los alumnos. Téngase en cuenta que dicho grupo se aprobó a finales de mayo de 2014, con los alumnos prácticamente en periodo de exámenes.

Los datos de la matrícula en el grupo de flipped classroom del primer cuatrimestre indican una composición de las asignaturas muy irregular, con asignaturas que se quedan en 4 alumnos y otra que alcanza los 14 .

Conviene añadir que estas cifras de matrícula crecieron moderadamente a medida que avanzaba el curso académico y en las asignaturas del segundo cuatrimestre la matrícula ha aumentado de forma notable.

Por último, la mayoría de los alumnos que optaron por el grupo de docencia inversa son repetidores y sólo se matricular de asignaturas sueltas dentro de este grupo. Esto significa que a la hora de hacer un seguimiento de los resultados del grupo de flipped classroom en la facultad de ADE existen ciertas dificultades debido a la heterogeneidad de los alumnos que lo componen. 


\section{Objetivos}

Se trata de determinar qué aspectos de los aplicados en el grupo de flip teaching de unaasignaturaconcreta, Derecho del Trabajo, pueden ser aplicados a grupos de docencia inversa en materias afines.

En otras palabras, qué puede aprovechar de la experiencia del grupo de docencia inversa de la asignatura de Derecho del Trabajo, un profesor de una asignatura de Derecho (o una materia afín) que tuviera que impartir su asignatura siguiendo esta metodología.

De este modo los objetivos concretos del presente estudio pueden exponerse en forma de preguntas:

¿Qué proporción de horas deben dedicarse a la clase presencial y al trabajo en casa ?

¿Qué tipo de materiales se han utilizado para que los alumnos puedan trabajar de forma autónoma?

¿Antes de una clase presencial sobre qué aspectos de un tema concreto debe trabajar el alumno?

¿Cómo debe diseñarse una clase presencial ? ¿Qué trabajar en una clase presencial?

¿Qué formas de control del trabajo autónomo del alumno van a establecerse? ¿Qué valor hay que dar a este control?

Visto el desarrollo del curso convendrá incidir en aquellos aspectos que se considera que han funcionado y que por lo tanto conviene mantenerlos para las siguientes ediciones del curso, y los aspectos sobre los cuales es necesario realizar algún tipo de modificación o, incluso directamente eliminar.

\section{Desarrollo de la innovación. Diseño de la asignatura.}

Vistos los principios que caracterizan la docencia inversa y establecido el marco en el que se inserta la asignatura Derecho del Trabajo, procede en este apartado, exponer qué se ha tenido en cuenta a la hora de diseñar dicha materia para impartirla de acuerdo con el citado modelo pedagógico.

\subsection{Descripción de la asignatura.}

La asignatura Derecho del Trabajo se encuadra dentro del segundo curso del Grado en ADE. Se imparte durante el primer cuatrimestre y tiene una carga docente de 6 créditos. De los cuales, 3 corresponden a teoría de aula, 2,6 a práctica de aula y 0,4 a práctica de campo, consistente esta última en la asistencia a un juicio laboral ante los juzgados de lo social de Valencia.

El objetivo fundamental de la asignatura de Derecho del Trabajo consiste en que el alumno conozca a un nivel básico y eminentemente práctico cuáles son los aspectos fundamentales

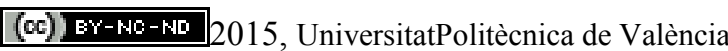

Congreso In-Red (2015) 
Docencia inversa aplicada a disciplinas jurídicas. Aplicación práctica en la asignatura de Derecho del Trabajo en el grado en $\mathrm{ADE}$.

del Derecho del Trabajo que se les van a aplicar en una eventual relación empresatrabajador.

Resulta obvio que, dadas las características del grado en el que se imparte la asignatura y de los alumnos a la que va dirigida, no se puede pretender que estos adquieran unos niveles jurídico-laborales exhaustivos como se exigiría a futuros profesionales del derecho, como abogados, jueces fiscales, etc... Va dirigida,en cambio, a manejar los conceptos teóricoprácticos básicos en Derecho Laboral propios de un futuro empresario. Por tanto, la asignatura pretende dar a conocer los principales conceptos jurídico-laborales, y su aplicación práctica desde el punto de vista empresarial.

En cuanto a los contenidos de la asignatura, interesa señalar que consta de 10 temas.

Cinco de los temas se consideran esenciales ya que sin los conceptos que en ellos se explican no sólo no se alcanzarían los objetivos de la asignatura sino que además son la base del resto de temas que sin aquellos no se entenderían. Otros tres temas se consideran necesarios ya que sin llegar al nivel de importancia de los citadas anteriormente son imprescindibles para alcanzar los objetivos de la asignatura.

Por último, otros dos temas tienen un carácter complementario de los anteriores lo cual no significa que deba prescindirse de ellos ya que inciden sobre temas relevantes en cualquier relación laboral pero sí que es cierto que pueden trabajarse de una forma independiente del resto de temas.

En lo que respecta a la distribución de la docencia en presencial y no presencial, ya se dijo en un apartado anterior que este reparto se había hecho siguiendo un criterio diferente del adoptado por el resto de profesores del grupo de docencia inversa.

En efecto, todos los profesoresdecidieron que la parte no presencial de sus respectivas asignaturas alcanzaría al $100 \%$ de la parte de teoría de aula quedando como presencial la parte de práctica de aula, práctica informática y seminario. Sin embargo, en lo que a la asignatura Derecho del Trabajo se refiere, la teoría de aula se imparte en el modelo no presencial en un $50 \%$ (y no en un 100\% como en el resto de asignaturas).

Esto se debe a que en dicha asignatura, como ya se ha mencionado, los primeros temas se consideran esenciales, de modo que si el estudiante no los retiene o encuentra dificultades en su comprensión corre el riesgo de no asimilar otros conceptos que se basan precisamente en estos, que son básicos.

Ante el temor de que esto ocurriera, ante la falta de experiencia en el uso del método de la clase inversa y ante la falta de tiempo para preparar pausadamente el curso, se optó por impartir los tres primeros temas de forma tradicional para introducir la flipped classroom a partir del temacuatro. Más adelante, en el apartado de resultados se valorará lo acertado o no de esta decisión.

\subsection{Diseño del material docente.}

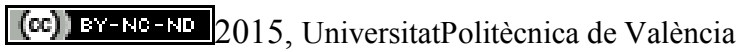


En lo que respecta al diseño del material docente, interesa plantear dos cuestiones clave. Primera, qué tipo de material debe ponerse a disposición de los alumnos para que cumpla con las funciones que éste debe cumplir (calidad, sencillez, utilidad ...) y que han sido expuestos en el punto primero de este escrito. Segunda, una vez decididos los soportes en que se va a presentar la documentación a los estudiantes conviene determinar los criterios que van a seguirse para decidir qué contenidos se presentan en un formato y qué contenidos se presentan en otro. Es decir, qué se va a presentar en formato audiovisual y qué no.

Abordando, la primera de las cuestiones planteadas, conviene recordar que se recomienda que el material que se proporcione al estudiante de la flipped classroom se contenga en formato audiovisual. Todo ello por las razones expuestas en el primer apartado de este escrito, resulta más atractivo al alumno, es más eficaz para el aprendizaje, facilita el trabajo autónomo, etc.

Por lo tanto, resulta evidente que conviene proporcionar parte de la docencia en este formato. Si al alumno se le entrega el material de la asignatura en material de lectura no verá ninguna diferencia entre el método de docencia inversa y el método tradicional y tenderá a buscar que le expliquen las conceptos correspondientes mediante la clase magistral que es lo que conoce.

Por lo tanto, hay que apostar por la conveniencia del uso del formato audiovisual en la clase inversa. ¿Pero en qué medida? ¿Cuánto debería ser en formato audiovisual? En mi opinión, proporcionar toda la docencia en video (por ejemplo, a través del programa de videoapuntes) resulta excesivo y monótono, y se corre el riesgo de provocar que el alumno desconecte y pierda el interés por esta metodología docente.

Lo conveniente, a mi juicio, es combinar una parte escrita con videos sobre aquellos contenidos que se consideren adecuados. En concreto, lo que se ha hecho en la asignatura de Derecho del Trabajo ha sido elaborar un resumen de los apuntes que tradicionalmente se ponía a disposición de los alumnos dejándolos prácticamente en un tercio o un cuarto de su extensión. Esta reducción en la parte escrita del tema se subsana introduciendo vínculos en el texto que enlazan con un video que exlplica el concepto en cuestión y que no figura por escrito.

En definitiva, el alumno dispone de unos apuntes muy reducidos por cada tema (entre 5 y 8 páginas) en los que determinadas cuestiones se explican por el docente a través de un video grabado al efecto. Aproximadamente se han grabado unos 5 o 6 videos con una duración por cada uno de ellos que ronda los 5 o 6 minutos.

Para la grabación de los correspondientes videos se ha utilizado la herramienta Screencast (existe al menos una versión gratuita fácilmente didponible en la red). Con ella se permite grabar de forma muy sencilla (en el propio ordenador del profesor) una combinación de

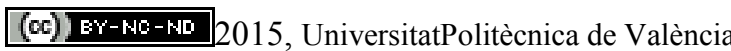

Congreso In-Red (2015) 
Docencia inversa aplicada a disciplinas jurídicas. Aplicación práctica en la asignatura de Derecho del Trabajo en el grado en $\mathrm{ADE}$.

imágenes y voz. En realidad, con esta herramienta el alumno puede visualizar en un video de 5 o 6 minutos las mismas diapositivas que sobre un tema determinado se proyectan durante una clase magistral y con los mismos (o similares) comentarios a los que se realizarían en dicha clase en ese mismo momento. Es como ver las diapositivas de clase con la explicación del profesor pero sin ver al docente.

En mi opinión, es una herramienta perfectamente válida para el objetivo que se persigue, produce videos con una calidad aceptable y tiene a su favor la comodidad que supone para el docente a la hora de grabar los videos.

Se ha dicho sobre esta herramienta que el alumno agradece ver a su profesor cuando le están explicando tal o cual concepto (como ocurre en los Polimedia). En mi opinión, esto que sería recomendable en un curso on-line no lo es tanto en el caso del flip teaching puesto que el estudiante recibe un trato directo por parte del profesor durante las clases presenciales.

Por último, conviene señalar que, junto con los apuntes confeccionados expresamente para la docencia inversa, los alumnos que siguen esta metodología también disponen, como los alumnos del resto de grupos, de los apuntes completos en formato de lectura y de las diapositivas completas que se proyectan durante las clase impartidas en formato magistral.

Una vez analizado el tipo de material docente que resulta más conveniente utilizar conviene decidir qué partes de los contenidos se presentan en formato y video y qué partes no. En otras palabra qué grabo y qué no grabo.

El primer criterio que se ha seguido es que lo más importante, lo básico de cada tema tiene que quedar muy claro y por lo tanto se presnta en formato de video. Los conceptos accesorios o complementarios de cada tema no es necesario presentarlos en dicho formato. Además, se da el caso de que determinados contenidos o incluso temas enteros no tienen un carácter básico o esencial pero resulta muy tediosa su explicación. Muchas veces se trata de enumeraciones de contenidos que no son díficiles de entender pero el hecho de leerlos o repetirlos en una clase magistral no aporta gran cosa, salvo aburrimiento general. Piénsese por ejemplo en la enumeración de las competencias de un órgano administrativo determinado. Si este contenido se le proporciona al alumno en formato de video podrá trabajarlo en el momento que él considere y le servirá de apoyo para retenerlo.

Si lo que acaba de exponerse se pone en relación con lo expuesto en el apartado anterior en el que se describe la asignatura Derecho del Trabajo, se da el caso de que dos de sus temas encajan perfectamente en lo que se ha llamado temas complementarios. Aplicando la metodología de docencia inversa, estos temas pueden estudiarse de forma totalmente autónoma por parte del alumno, pudiendo el profesor centrarse en otros contenidos que requieren mayor práctica o atención debido a su complejidad.

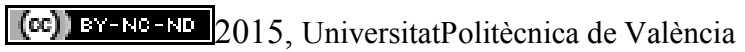


Carlos García Gallego

\subsection{Diseño de las actividades de aula}

El segundo pilar sobre el que se asienta la docencia inversa es el correcto diseño de las actividades en el aula. Esto supone un trabajo previo de planificación del profesor,que al programar la asignatura, debe concretar unas actividades que sean coherentes con los contenidos trabajados por el alumno de forma autónoma y adecuadas a los mismos.

Básicamente las sesiones presenciales que se han llevado a cabo en la asignatura de Derecho del Trabajo pueden dividirse en tres fases.

En primer lugar, durante los diez o quince primeros minutos de la clase se realiza por parte del profesor un repaso de los conceptos que tenían que haberse trabajado en casa por parte de los estudiantes. Si existen dudas o preguntas al respecto se resuelven.

A continuación, se plantea a los alumnos alguna actividad que permita aplicar los contenidos trabajados de forma autónoma. Normalmente estas actividades suelen consistir en la resolución de casos, en ocasiones de forma individual y en ocasiones de manera colectiva. Las actividades prácticas que se realizan van aumentando en complejidad a medida que se avanza en cada el tema y en el conjunto del curso.

Por último, antes de finalizar la clase presencial se corrige el supuesto o los supuestos que se hayan planteado y se resuelven las dudas que pudiera haber.

\subsection{Evaluación y tutorías.}

Conviene señalar, que los contenidos de la asignatura de Derecho del Trabajo en su modalidad de flipped classroom y en su modalidad presencial son exactamente los mismos y por lo tanto el trabajo que debe exigirse al alumno debe ser también idéntico en ambos casos. Lo que ocurre es que la distribución de ese trabajo será distinta según la modalidad docente que se siga. Así, al alumno de la clase inversa se le pide un trabajo mucho más constante y regular que al estudiante de una clase tradicional que suele concentrar sus esfuerzos en la fase de evaluación.

Dicho esto, la evaluación de la asignatura tiene que ser igualmente la misma cualquiera que sea el método pedagógico que se aplique en la asignatura.

En el caso de Derecho del Trabajo, los alumnos son evaluados mediante dos pruebas : la primera, que suele producirse a mediados (o finales) del mes de octubre, es una evaluación escrita teorico-práctica sobre los cuatro primeros temas de la asignatura. Y la segunda, que tiene lugar en el mes de diciembre, es muy similar a la anterior con la peculiaridad de que

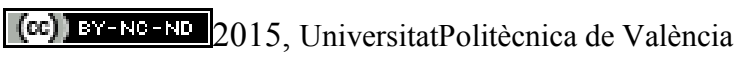

Congreso In-Red (2015) 
Docencia inversa aplicada a disciplinas jurídicas. Aplicación práctica en la asignatura de Derecho del Trabajo en el grado en $\mathrm{ADE}$.

abarca los 6 temas siguientes. Como se ha dicho, esta evaluación es común a todos los alumnos de la asignatura.

No obstante, un $10 \%$ de la calificación final de los alumnos del grupo de docencia inversa está constituida por la nota que regularmente van recibiendo por realizar correctamente las actividades de control del trabajo autónomo. Conceder este $10 \%$ es crucial (y en el caso que nos ocupa, suficiente) para conseguir que el estudiante mantenga esa línea de trabajo en casa imprescindible para el correcto funcionamiento del método de flip teaching.

En lo que respecta a las tutorías, no hay ninguna diferencia entre el régimen establecido en el grupo de flipped classroom y el tradicional. En ambos casos la tutorías son a demanda.

No se ha observado un incremento especial en la solicitud de tutorías por parte de los alumnos de flip teaching respecto al resto de grupos, lo cual parece lógico teniendo en cuenta que durante las sesiones presenciales se dedica una parte del tiempo a resolver dudas.

\section{Resultados.}

Los resultados obtenidos en el grupo de docencia inversa pueden clasificarse en dos apartados perfectamente diferenciados.

Por una parte, habría que analizar los resultados en relación con el docente, y por otra en segundo lugar, los resultados desde el punto de vista de los alumnos.

Desde el punto de vista del profesor los resultados pueden considerarse, en términos generales, como bastantes satisfactorios. En particular cabría destacar lo siguiente:

- Debido a que se trata de un modelo docente novedoso, que requiere de una preparación específica de material docente y de una planificación minuciosa de las actividades de aula, el grupo de docencia implica un trabajo importante del docente para ponerlo en marcha. A esto hay que añadir que en el caso concreto de la asignatura Derecho del Trabajo el tiempo de preparación de que se dispuso fue muy escaso.

En cualquier caso, ese esfuerzo de preparación se amortiza, por así decirlo, en el plazo de un curso académico. A partir de entonces lo más complicado en el desarrollo de este grupo ya estaría hecho.

- La estrategia seguida en la asignatura en Derecho del Tarbajo según la cual los tres primeros temas se impartía de forma tradicional y a partir del tema 4 se seguía con el modelo de flipped classroom es totalmente errónea y nada recomendable.

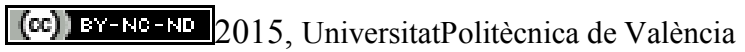

Congreso IN-RED (2015) 
En efecto, recuérdese que se optaba por este sistema para garantizar que los alumnos asimilaran unos conceptos que se consideran básicos en la asignatura. Dichos conceptos efectivamente se asimilaron, pero podrían haberse asimilado perfectamente siguiendo el modelo de docencia inversa.

Lo que ocurrió fue que mientras se impartían los primeros temas según el modelo de clase magistral se perdió un tiempo precioso para que los alumnos asimilaran el modelo de flip teaching. De este modo, el citado modelo tardó más en implantarse correctamente en el grupo.

- Es fundamental para el funcionamiento de la docencia inversa que los alumnos realicen la parte del trabajo autónomo que les corresponde. Al principio del curso cuesta un poco que lo lleven a cabo puesto que no están habituados al nuevo sistema. Es ahí donde el profesor debe insistir y mantenerse firme en que dichas tareas se realicen. Si esto se consigue, los alumnos se acostumbran al nuevo método y ya no es tan necesario insistirles.

- Si el material que se pone a disposición de los alumnos es bueno, si las tareas presenciales se programan adecuadamente y si se consigue que los alumnos realicen su parte de trabajo en casa, la docencia mediante el flip teaching es mucho más cómoda que la docencia tradicional. Cosa lógica puesto que la gran parte de ese trabajo ya está hecho de antemano. En especial, esto se nota mucho cuando el profesor tiene que dar la misma asignatura a distintos grupos.

Desde el punto de vista del alumno los resultados pueden resumirse en las cosideraciones, siguientes:

- Las calificaciones obtenidas en el grupo de docencia inversa son perfectamente equiparables a los obtenidos en el resto de grupos de la asignatura que siguen el método tradicional. Es más, en el segundo parcial, en el que todos los temas se impartieron de acuerdo con el método de la flipped classroom los alumnos obtuvieron en general mejores resultados que en el primero.

Además, una vez terminado el primer parcial, varios alumnos del resto de grupos de la asignatura que habían suspendido se cambiaron de grupo matriculándose en el de docencia inversa. Todos ellos aprobaron el segundo parcial y la recuperación del primero.

- Los materiales proporcionados a los alumnos, y en particular los videos grabados mediante screencast fueron muy bien acogidos por ellos. No hubo queja alguna en cuanto a la calidad de la imagen o del sonido.

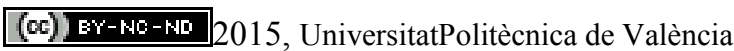


Docencia inversa aplicada a disciplinas jurídicas. Aplicación práctica en la asignatura de Derecho del Trabajo en el grado en $\mathrm{ADE}$.

- Las dificultades que los alumnos podían presentar en relación con los contenidos de la asignatura se detectan de forma rápida y eficaz. Probablemente en esto influye bastante el hecho de tratarse de un grupo muy reducido de estudiantes.

- En general las opiniones que los alumnos han vertido sobre el modelo son positivos. Parece, según la experiencia de la facultad de ADE que es especialmente atractivo para los alumnos repetidores.

\section{Conclusiones.}

Las conclusiones van dirigidas a determinar si puede resultar aplicable la experiencia ofrecida en el presente trabajo a otras disciplinas jurídicas o a cualquier área relacionada con las ciencias jurídicas o humanidades. Y de ser así, en qué aspectos puede serlo.

En principio la respuesta debe ser claramente afirmativa. En mi opinión, si al alumno se le proporcionan materiales de calidad, ajustados a los objetivos que se persiguen en la asignatura, se planifica adecuadamente la actividad presencial en el aula y sobre todo, si se consigue establecer un sistema eficaz de control del trabajo autónomo del alumno, el modelo es perfectamente aplicable, a cualquier disciplina jurídica.

Se ha criticado este modelo en relación con su aplicación a asignaturas de derecho en el sentido de que que se perdería la tarea que hace el docente de interpretación y aplicación de las normas que asimila el alumno. Sobre este particular interesa indicar que con la docencia inversa el trabajo del profesor no desaparece. Al contrario, tanto la parte interpretativa del Derecho como su aplicación práctica se trabajan durante la clase presencial (o debería), de modo que ese aspecto de la docencia de las disciplinas jurídicas esta perfectamente cubierto. Incluso más que en un grupo tradicional.

También se ha dicho que el modelo de flipped classroom no sería aplicable a disciplinas jurídicas dado el carácter cambiante de las mismas. En otras palabras, cada vez que se produce un cambio normativo en una materia habría que cambiar el temario y eso resultaría especialmente gravoso en el caso de que se utilizara el modelo de clase inversa.

En realidad, los cambios normativos influyen en los contenidos de las asignaturas jurídicas sea cual sea el modelo docente que quiera aplicarse. Si el cambio normativo es superficial o accesorio (lo que suele ser frecuente) los cambios en la asignatura también lo serán y si el cambio en la ley es profundo (cosa menos habitual) afectara de igual modo al contenido de la asignatura.

Después de todo lo dicho, la principal incertidumbre que, a mi juicio, planea sobre este tipo de metodología es si este modelo puede ser aplicado con la misma eficacia a grupos más grandes, de entre 60 y 80 alumnos

(c) EY-NC-ND 2015, UniversitatPolitècnica de València 
Carlos García Gallego

\section{Referencias.}

BERGMANN, J. y SAMS, A., (2013). Dale la vuelta a tu clase. Madrid: SM.

SANTIAGO, R., TOURON, J. y DÍEZ, A. (2014). The Flipped Classroom: Cómo convertir la escuela en un espacio de aprendizaje. Barcelona: Digital-Text. 